\title{
Análise dos processos erosivos e deposicionais utilizando o modelo SIMWE (Simulated of Water Erosion)
}

\author{
Erosion and Deposition Process assessment using SIMWE model
}

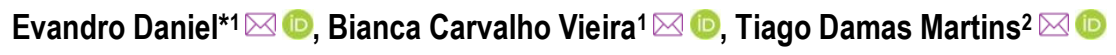

1Departamento de Geografia, Faculdade de Filosofia Letras e Ciências Humana, Universidade São Paulo, São Paulo, SP, Brasil

Recebido (Received): 15/05/2021

2Instituto das Cidades, Universidade Federal de São Paulo, São Paulo, SP, Brasil Aceito (Accepted): 06/10/2021

E-mail: biancacv@usp.br (BVC); td.martins@unifesp.br (TDM)

*E-mail para correspondência: evandrogeo@usp.br

Resumo: Nos últimos anos houve um avanço no uso de modelos matemáticos para avaliação da dinâmica espaço-temporal dos processos erosivos. Entre estes, destaca-se o SIMWE (Simulated of Water Erosion), que é um modelo dinâmico, de base física, projetado para ambiente temperado, capaz de simular a erosão, o transporte e a deposição por fluxo superficial, podendo ser aplicado em situações complexas do terreno, solo e uso. Neste sentido, este trabalho teve como objetivos centrais avaliar a aplicação do modelo SIMWE para ambiente tropical a partir da análise dos processos erosivos e deposicionais na bacia do Alto Espraiado - São Pedro (SP) e, assim, analisar a influência dos parâmetros utilizados na geração de cenários de erosão/deposição. Foram simulados seis cenários considerando distintos valores de intensidades pluviométricas; taxa de excesso de precipitação, taxa de infiltração, permeabilidade, tensão de cisalhamento, coeficiente de Manning. O produto final do Balanço da Erosão e de Deposição aponta que grande parte da área foi classificada como áreas deposicionais (valores negativos) e erosivas (valores positivos), sobretudo em margens das drenagens e curvaturas côncavas, justamente onde estão situadas as feições erosivas mapeadas e também onde foram registrados os maiores valores dos fluxos de sedimentos e hidrológicos superficiais. Tais resultados demonstraram a viabilidade e a sensibilidade da aplicação do SIMWE em ambiente tropical como uma ferramenta importante para diagnóstico e prognóstico em relação à questão do uso e ocupação do solo.

Palavras Chaves: Modelagem de Erosão Hídrica; Geoprocessamento; GRASS.

Abstract: In recent years, there has been an advance in the use of mathematical models to assess the spatial temporal dynamics of erosive processes. Among these, the SIMWE (Simulated of Water Erosion) stands out as a dynamic physically-based model designed for a temperate environment, capable of simulating erosion, transport, and deposition by surface flow, which can be applied in complex terrain, soil, and land use. This work aimed to evaluate the use of the SIMWE model for the tropical environment based on the analysis of erosive and depositional processes in the Alto Espraiado basin - São Pedro (SP), as well as to assess the influence of the parameters used in the generation of scenarios erosion/deposition. Six scenarios were simulated considering different values of rainfall intensities; excess precipitation rate, infiltration rate, permeability, shear stress, Manning coefficient. The final product of the Balance of Erosion and Deposition points out that a large part of the area was classified as deposition (negative values) and erosion (positive values) on the banks of the drainages and concave curvatures, precisely where the mapped erosive features are located and where they have recorded the highest values of sediment and surface hydrological fluxes. These results demonstrate the feasibility and sensitivity of applying SIMWE in a tropical environment and as an important tool for diagnosis and prognosis about the issue of land use and occupation.

Keywords: Water Erosion Modeling; Geoprocessing; GRASS. 


\section{Introdução}

Os processos erosivos estão associados à degradação dos solos e são considerados como um problema mundial, pois são responsáveis por cerca de $80 \%$ de todas as terras degradadas do planeta (MA et al., 2021). No Brasil, a maioria dos municípios paulistas, nos contextos urbanos ou rurais, sobretudo em coberturas pedológicas mais arenosas, apresentam feições erosivas que provocam a perda de solos e o assoreamento de reservatórios e cursos d’água (ALMEIDA FILHO et al., 2016).

Para aprimorar a avaliação dos processos erosivos e da conservação do solo diferentes métodos têm sido desenvolvidos, como a Equação Universal de Perda de Solo (USLE), que permite a quantificação das taxas de perda de solos provenientes da erosão laminar e por sulcos durante um determinado período, a partir de parcelas experimentais e de parâmetros de erosividade, erodibilidade, topográficos, cobertura vegetal e práticas de manejo (WISCHMEIER; SMITH, 1978). Contudo, esse método possui limitações em condições diferentes de sua proposição de origem (BERTONI; LOMBARDI NETO, 2012), devido à necessidade de um grande volume de dados de entrada; por desconsiderar alguns processos físicos como o desprendimento e o transporte de partículas do solo e não predizer a distribuição espacial e temporal da erosão hídrica, como também a deposição e o aporte de sedimentos (FERNANDES, 1997; CARVALHO, 2014).

No sentido de corrigir e superar as limitações da USLE surgiram outras versões: a RUSLE (Revised Universal Soil Loss Equation) (RENARD et al., 1997) e a MUSLE (Modification Universal Soil Loss Equation) (WILLIAMS, 1975). Entretanto, as restrições dessas alterações também são bastante discutidas devido à dependência de dados gerados em parcelas de erosão e à desconsideração do processo de deposição dos sedimentos, o que dificulta a sua aplicação em maiores escalas (PINTO, 2014).

A RUSLE possui a mesma estrutura da equação da USLE, porém com algumas mudanças, como o auxílio de um programa computacional que facilita a estimativa da perda de solo (NEARING et al., 2005; LEITE, 2009). Tal modelo avalia a perda média anual de solo associada à precipitação (em um período de vinte anos) e ao escoamento, considera conceitos da modelagem da erosão com base na descrição do processo físico como forma de melhorar as predições de erosão e, ainda analisa as perdas de solos provocadas pelas erosões laminares e em sulcos (AMORIM, 2004). As limitações principais estão associadas à base empírica que dificulta muito sua aplicação para outras condições edafoclimáticas e o fato de desconsiderar o processo de deposição, o que limita a sua aplicação para grandes áreas (PINTO, 2014).

Em relação à MUSLE trata-se de uma equação modificada da Equação Universal de Perda de Solo (EUPS) proposta e desenvolvida por Wischmeier e Smith $(1965 ; 1978)$, a qual calcula a média anual bruta da erosão em função da substituição do fator energia cinética da chuva pelo escoamento e vazão (WILLIAMS, 1975). Tal modelo, melhora a projeção e a estimativa da produção de sedimentos, pois considera o escoamento em substituição a chuva e permite que a equação seja aplicada para eventos individuais de precipitação (AVANZI et al., 2008).

O modelo WEPP (Water Erosion Prediction Project), de Flanagan e Nearing (1995), determina as taxas de erosão e de deposição de sedimentos (diário, mensal ou anual), a partir de equações relacionadas às teorias de infiltração, física do solo, hidráulica, mecânica da erosão e fisiologia vegetal. Este modelo, diferente dos demais, considera os efeitos das mudanças de uso do solo e modela a variabilidade espacial e temporal na topografia, rugosidade da superfície, propriedades do solo e culturas (FLANAGAN et al., 2007; PEREIRA, 2014).

O modelo SIMWE (MITAS e MITASOVA, 1998) simula a erosão, o transporte e a deposição por fluxo superficial. Seus parâmetros de entrada consistem no modelo de erosão de encostas (WEPP), que define as condições iniciais do processo de erosão/deposição e possibilita ser aplicado em situações complexas do terreno, solo e uso (KOCO, 2011; HOFIERKA; KNUTOVA, 2015).

A estimativa da erosão por meio de modelos em bases físicas permite examinar diferentes cenários a partir de simulações, sendo uma importante ferramenta de estudo da dinâmica do processo. Neste sentido, este estudo teve como objetivos centrais avaliar a aplicação do modelo SIMWE em ambiente tropical úmido por meio da dinâmica dos processos erosivos e deposicionais no município de São Pedro (SP) e analisar a influência dos parâmetros utilizados na geração de cenários de erosão/deposição.

\section{2. Área de estudo}

Para análise das feições erosivas e deposicionais foi utilizada uma bacia experimental (bacia do Alto Espraiado) com cerca de $2 \mathrm{~km}^{2}$, situada ao norte do município de São Pedro (SP) (Figura 1). Seu canal principal deságua no Ribeirão Araquá, afluente do Rio Piracicaba, com alta densidade de feições erosivas 
lineares (Figura 2), em diversos estágios e com áreas entre $300 \mathrm{~m}^{2}$ até $48.000 \mathrm{~m}^{2}$ e profundidades variando entre $6 \mathrm{~m}$ e $22 \mathrm{~m}$ (DANIEL; VIEIRA, 2015).

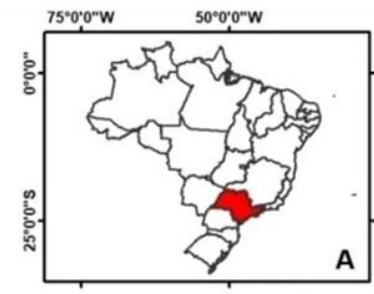

$47^{\circ} 54^{\circ} 0^{\prime \prime} \mathrm{W}$

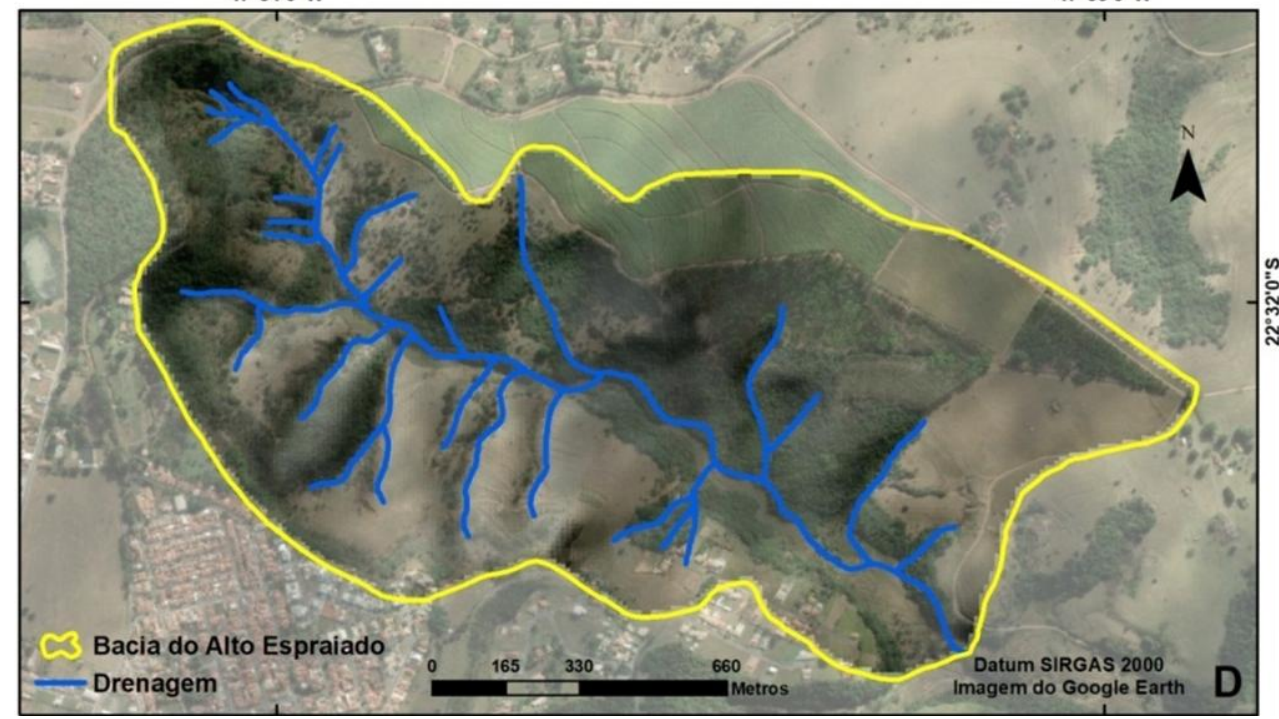

Figura 1: (A) Localização do estado de São Paulo no território brasileiro. (B) Posição do município de São Pedro no estado de São Paulo. (C) Bacia do Alto Espraiado no município de São Pedro. (D) Bacia do Alto Espraiado. Elaborada pelos autores.

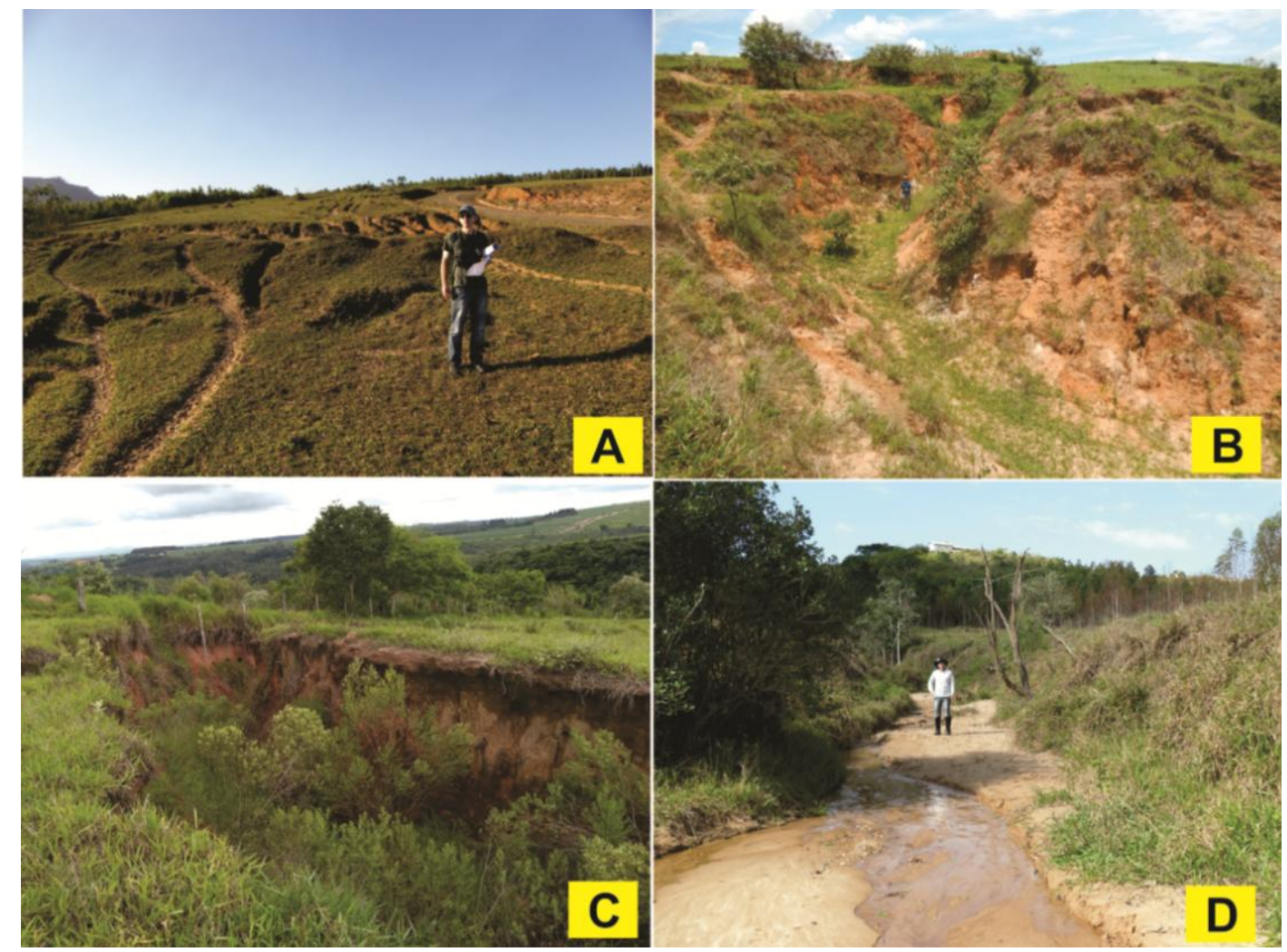

Figura 2: Registro de processos erosivos na bacia do Alto Espraiado - São Pedro (SP). (A) Sulcos erosivos. (B) Feições erosivas lineares avançadas - voçorocas. (C) Feições erosivas intermediárias - ravinas. (D) Registro de assoreamento em afluente da bacia do Alto Espraiado. Fonte: Autores (2020). 
A seleção desta bacia se deu diante da disponibilidade de dados para o emprego do SIMWE, como valores diários de precipitação, além de dados coletados diretamente no campo referente aos solos, litologia e morfometria, por meio de pesquisas anteriores, tais como: Rodrigues (1982), Santoro (1991), Santos e Fulfaro (1996), Gomes (2002), Ferreira (2004), Mathias et al., (2013), Daniel e Vieira (2015).

A área está situada sobre a Formação Pirambóia (Grupo São Bento) pertencente à Bacia Sedimentar do Paraná e inserida na Depressão Periférica Paulista, compartimento de origem denudacional, embutido entre áreas serranas elevadas e acidentadas $(750-1600 \mathrm{~m})$ e as escarpas e festões das altas cuestas basálticas $850 \mathrm{~m}$ - 1100m (AB`SABER, 1949; SANCHEZ, 1969).

O solo é marcado pela presença de horizonte subsuperficial arenoso e espesso, oriundo de rochas arenosas, com bandas onduladas intercalares e lentes arenosas eluviadas, passando na base dos perfis, a um horizonte profundo, com o predomínio conforme a nova classificação da Embrapa (1999) de Neossolo Quartzarênico (SANCHEZ, 1971; KAWAKUBO et al.,2006). A constituição pedológica apresenta registros de Neossolo Litólico e Gleissolo e Neossolo Quartzarênico em grande parte da área, Neossolos Flúvicos e Gleissolos ao longo do canal principal e Latossolo Vermelho na área circundante a montante do canal principal (FURQUIM, 2002).

A pluviosidade média anual varia entre 1200 e $1370 \mathrm{~mm}$, excetuando o ano de 1983 que registrou $2200 \mathrm{~mm}$ e os anos de 1960, 1963, 1968 e $1982 \mathrm{com}$ montantes menores que $1000 \mathrm{~mm}$. Destaque para o período chuvoso nos primeiros e nos últimos meses do ano (verão), e um período sensivelmente mais seco (inverno seco) nos demais meses, aproximadamente 30 mm (CONTI, 1971; SANTORO, 1991; CAPELLA et al.,2021).

$\mathrm{O}$ uso do solo da bacia do alto Espraiado encontra-se, de forma geral, destinado às práticas de pastagens (pasto sujo e pasto limpo), áreas agrícolas, silvicultura, a mata em setores circundantes dos afluentes e da drenagem principal, áreas côncavas e parte superior de algumas encostas e, por fim a presença de solo exposto, também em vertentes côncavas e nas áreas urbanas (DANIEL et al., 2021).

\section{Materiais e métodos}

\subsection{Modelo SIMWE}

O modelo SIMWE está estruturado em dois módulos (Figura 3), sendo um de "fluxos hidrológicos superficiais", que simula o escoamento superficial com base nas variáveis espaciais de precipitação e de solos; e o módulo de "fluxos de sedimentos", que calcula a erosão do solo, o transporte e a deposição de sedimentos originados pelo fluxo de água (NETELER; MITASOVA, 2008). Sua operação pode ser feita por meio do GRASS (Geographic Resources Analysis Support System) (GRASS, 2017) e as execuções são realizadas com base em um Modelo Digital de Elevação (MDE), que possibilita a simulação dos fluxos de água e sedimentos (MITAS; MITASOVA, 1998).

Módulo - Fluxos Hidrológicos (r.sim.water)

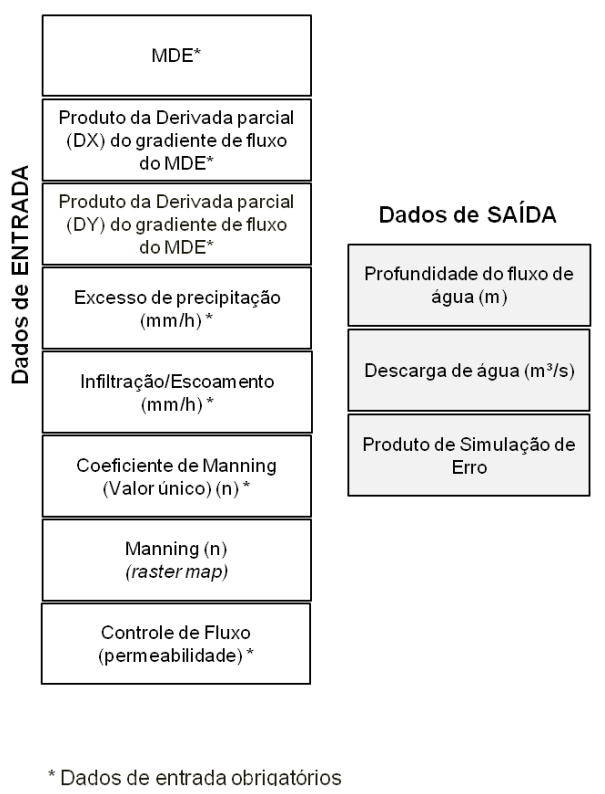

Módulo - Fluxos de Sedimentos (r.sim.sediment)

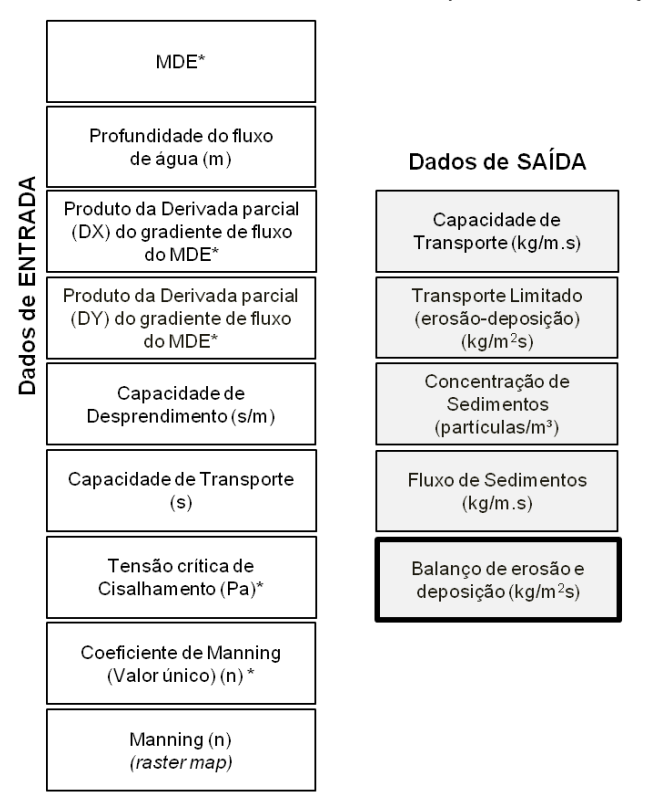

Figura 3: Estrutura dos módulos "fluxo hidrológico superficial" e "fluxo de sedimentos" do SIMWE. Fonte: Adaptado de Fernandes (2014). 
Além do MDE devem ser selecionados parâmetros hidrológicos, pedológicos e geotécnicos, tais como Taxa de Excesso de Precipitação $(\mathrm{mm} / \mathrm{h})$, Coeficiente de Manning (n), Taxa de Infiltração $(\mathrm{mm} / \mathrm{h})$, Permeabilidade $(\mathrm{cm} / \mathrm{s})$, Coeficiente da Capacidade de Desprendimento $(\mathrm{s} / \mathrm{m})$, Coeficiente da Capacidade Transporte de Sedimentos (s) e Tensão de Cisalhamento (Pa) (Tabela 1).

Tabela1: Parâmetros empregados no SIMWE, conceitos principais e os valores considerados nesta pesquisa.

\begin{tabular}{cc}
\hline $\begin{array}{c}\text { PARÂMETRO } \\
\text { (unidade) }\end{array}$ & CONCEITO (Fonte Bibliográfica) \\
& \\
Taxa de Excesso de & $\begin{array}{c}\text { Volume de água disponível para o escoamento. } \\
\text { Estimada a partir da intensidade da precipitação } \\
\text { e da taxa de infiltração }(\mathrm{mm} / \mathrm{h} \text { ) }\end{array}$ \\
Precipitação $(\mathrm{mm} / \mathrm{h})$ & (MITASOVA et al., 1997; WARREN, 1998)
\end{tabular}

\section{VALORES UTILIZADOS NESTA PESQUISA (Fonte Bibliográfica)}

190 e 87 (acumulado de 7 dias). (CIIAGRO, 2021)

Considera-se o excesso de precipitação acima de $60 \mathrm{~mm} / \mathrm{h}$ como potencialidade erosiva

(SANTOS et al., 2010; HAO et al., 2019)

Coeficiente de Manning
(n)

Rugosidade da superfície, em que os baixos valores indicam rugosidade mínima e os altos valores referem-se à rugosidade significativa. (WARREN, 1998)

Está associada à condutividade hidráulica

Taxa de Infiltração $(\mathrm{mm} / \mathrm{h})$ (NETELER; MITASOVA, 2008; MITASOVA et al., 2013)

Indica a facilidade da água percolar no solo. Equivalente a velocidade de fluxo sob gradiente unitário (FERNANDES, 2014)

Permeabilidade $(\mathrm{cm} / \mathrm{s})$

Coeficiente da

Capacidade de

Desprendimento ( $/ \mathrm{m})$

Coeficiente da Capacidade Transporte de Sedimentos (s)

Associado à erodibilidade. Destacamento do solo por unidade ao aumento da tensão de cisalhamento do fluxo de água (FLANAGAN; NEARING, 1995)

Fluxo de sedimentos, a capacidade do solo ser transportado pelo fluxo de água. Depende das propriedades do solo e da influência da vegetação (WARREN, 1998)

Tensão de Cisalhamento (Pa)

Resistência (propriedade e cobertura) de um solo às forças de cisalhamento. (FLANAGAN; NEARING, 1995)
0,013 (trechos de galeria pluvial)

(MATHIAS et al., 2013) e 0,079 (pastagem)

(MINGOTI, 2012)

Mínimo: 4 e Máximo: 12 (GOMES, 2002)*

Mínimo: 0,00024 e Máximo: 0,00488 (SANTORO e FÚLFARO, 1996; FERREIRA, 2004)**

0,6 (velocidade mínima) e 0,7 (velocidade máxima) - Solos Arenosos (RODRIGUES, 1982; SILVA, 1999)

Velocidade Mínima: 0,6 e Velocidade Máxima: 0,7 (RODRIGUES, 1982**; SILVA, 1999)

11000 (mínimo) e 18000 (máxima) (FERREIRA, 2004)***

* Infiltrômetro de Anel Duplo (IAD); **Permeâmetro de Carga Constante e Permeâmetro de Guelph; ***Ensaio de Compactação Proctor Normal.

\subsection{Modelo Digital de Elevação (MDE)}

A partir do MDE foram extraídos parâmetros morfométricos do relevo (declividade, aspecto e as derivadas parciais (dx e dy) do campo de elevação), utilizados para o cálculo do escoamento superficial, da direção e da magnitude do fluxo de água. O MDE foi elaborado a partir das curvas de nível representadas na carta topográfica São Pedro I (SF-23-Y-A-IV-1-NO-B) em escala 1:10.000 da Terra Foto (1979). Esses dados foram empregados na ferramenta topo to raster, apoiada na proposta de Hutchinson (1988). Também foi aplicada a ferramenta Sinks e posteriormente Fill para o preenchimento dos vazios e correções (MARK, 1988; TARBOTON et al.,1991). A resolução espacial do raster de 10 metros foi definida a partir da proposição de Hengl (2006), método conhecido como complexidade do relevo.

\subsection{Parâmetros de Entrada e Simulação dos Cenários}

Para a análise da influência das propriedades hidrológicas e do solo no desenvolvimento dos processos de erosão e de deposição, foram considerados e selecionados dados pluviométricos e pedológicos variáveis, totalizando seis cenários. O cenário 1 visou simular uma paisagem com maior suscetibilidade (maior valor para taxa de excesso de precipitação, menores valores para o coeficiente de Manning, a taxa de infiltração e permeabilidade). Os cenários 2, 3, 4 visaram simular dados intermediários e nos cenários 5 e 6 foram 
desconsiderados os seguintes parâmetros: tensão de cisalhamento, coeficiente de capacidade de desprendimento e capacidade de transporte de sedimentos (Tabela 2).

Tabela 2: Cenários com os respectivos dados de entrada para cada parâmetro do modelo SIMWE.

\begin{tabular}{ccccc|ccc}
\hline MÓDULO & \multicolumn{3}{c|}{ Fluxo de Água- r.sim.water - (unidade) } & \multicolumn{3}{c}{ Fluxo de Sedimentos- r.sim.sediment - } \\
(unidade)
\end{tabular}

Fonte dos dados: Rodrigues (1982); Santoro e Fúlfaro (1996); Silva (1999); Gomes (2002); Ferreira (2004); Mingoti (2012); Mathias et al., (2013); CIIAGRO (2021).

Para validar os cenários foi utilizado um mapa de feições erosivas elaborado por Daniel e Vieira (2015) (Figura 4). Os autores mapearam as feições a partir de ortofotos e de levantamentos de campo, considerando alguns critérios visuais como: sombreamento, solo exposto, entalhamento do relevo, fissuras no solo e declividade acentuada. Foram mapeados 14 polígonos de feições erosivas de diferentes tamanhos (entre $335 \mathrm{~m}^{2}$ e $48.599 \mathrm{~m}^{2}$ ) em estágios evolutivos intermediários (ravinas) e avançados (voçorocas). Posteriormente, a partir destes mapeamentos, aplicou-se a ferramenta Tabulate Intersection do programa ArcGis 10.5, para correlacionar quantitativamente entre as feições erosivas e os intervalos dados pelo SIMWE do balanço da erosão e deposição.

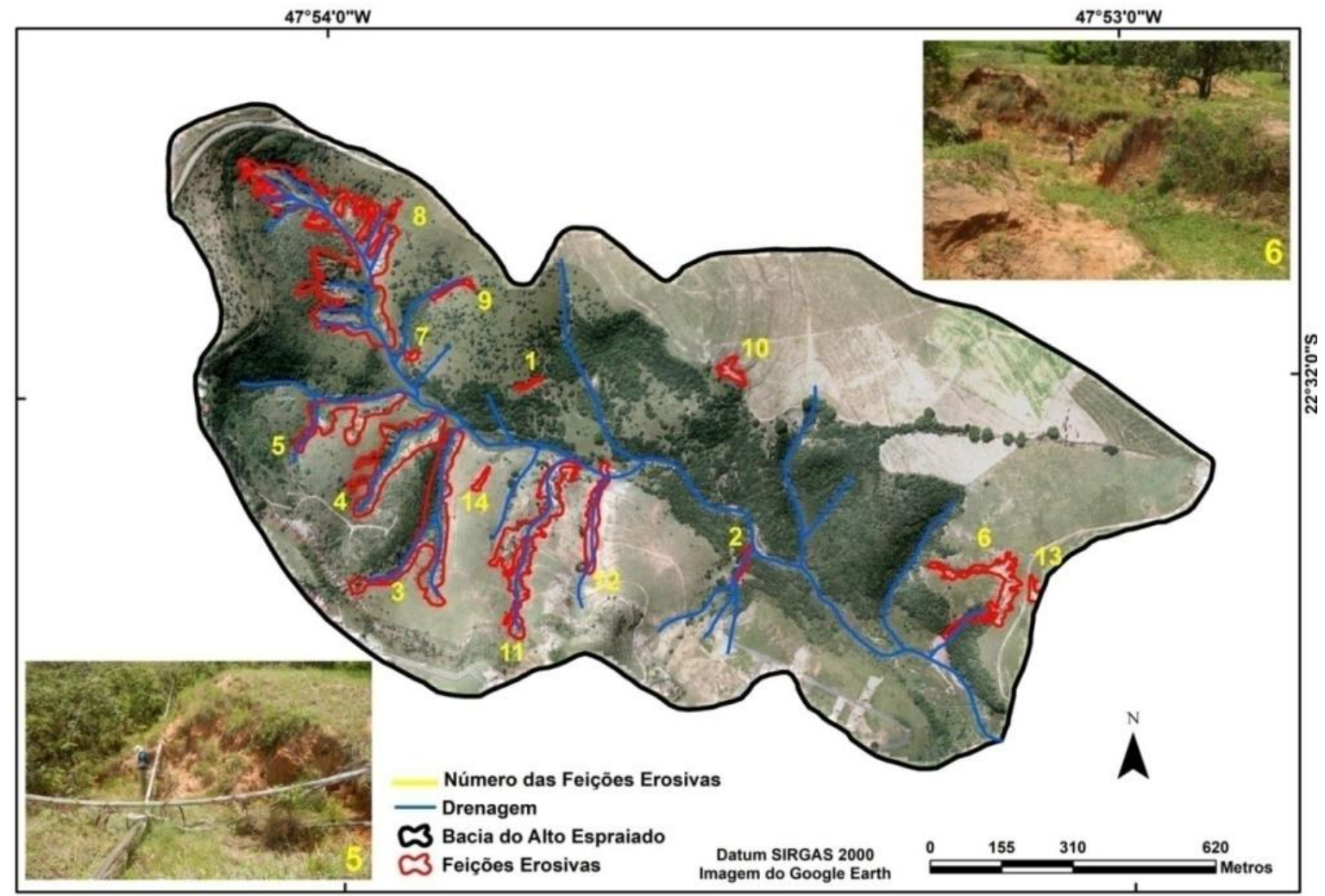

Figura 4: Mapa das feições erosivas da Bacia do Alto Espraiado, com destaque para algumas feições erosivas (pontos 5 e 6). Fonte: Adaptado de Daniel e Vieira (2015). 


\section{Resultados e discussões}

O valor mínimo registrado para deposição foi de $-1,17\left(\mathrm{~kg} / \mathrm{m}^{2} \mathrm{~s}\right)$ e o valor máximo de $-80,68\left(\mathrm{~kg} / \mathrm{m}^{2} \mathrm{~s}\right)$. Já os valores de erosão variaram entre $1,17\left(\mathrm{~kg} / \mathrm{m}^{2} \mathrm{~s}\right)$ até $80,68\left(\mathrm{~kg} / \mathrm{m}^{2} \mathrm{~s}\right)$ (Figura 5). Os resultados obtidos pelo SIMWE nos quatro primeiros cenários (1 a 4) apresentaram semelhanças na distribuição espacial dos processos de erosão/deposição. As simulações com os distintos valores dos parâmetros resultaram em variações nos valores de erosão (positivo) e de deposição (negativo) para os cenários 1 a 4 , porém a distribuição espacial destes processos se manteve bastante similar entre os cenários. Os maiores valores de deposição foram registrados em áreas circundantes aos canais de drenagem e em setores de curvaturas côncavas (Figura 5).
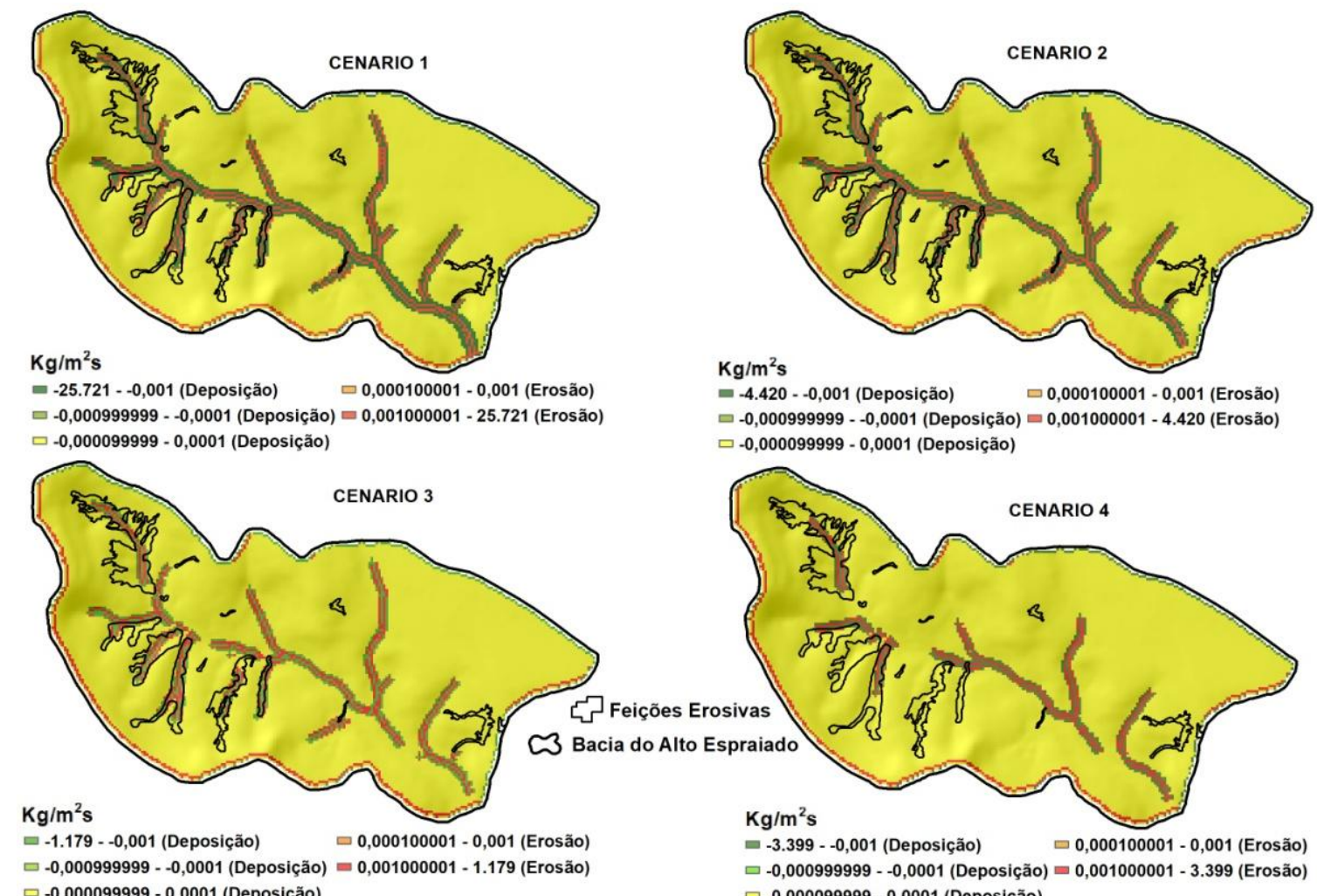

- $-0,000099999-0,0001$ (Deposição)
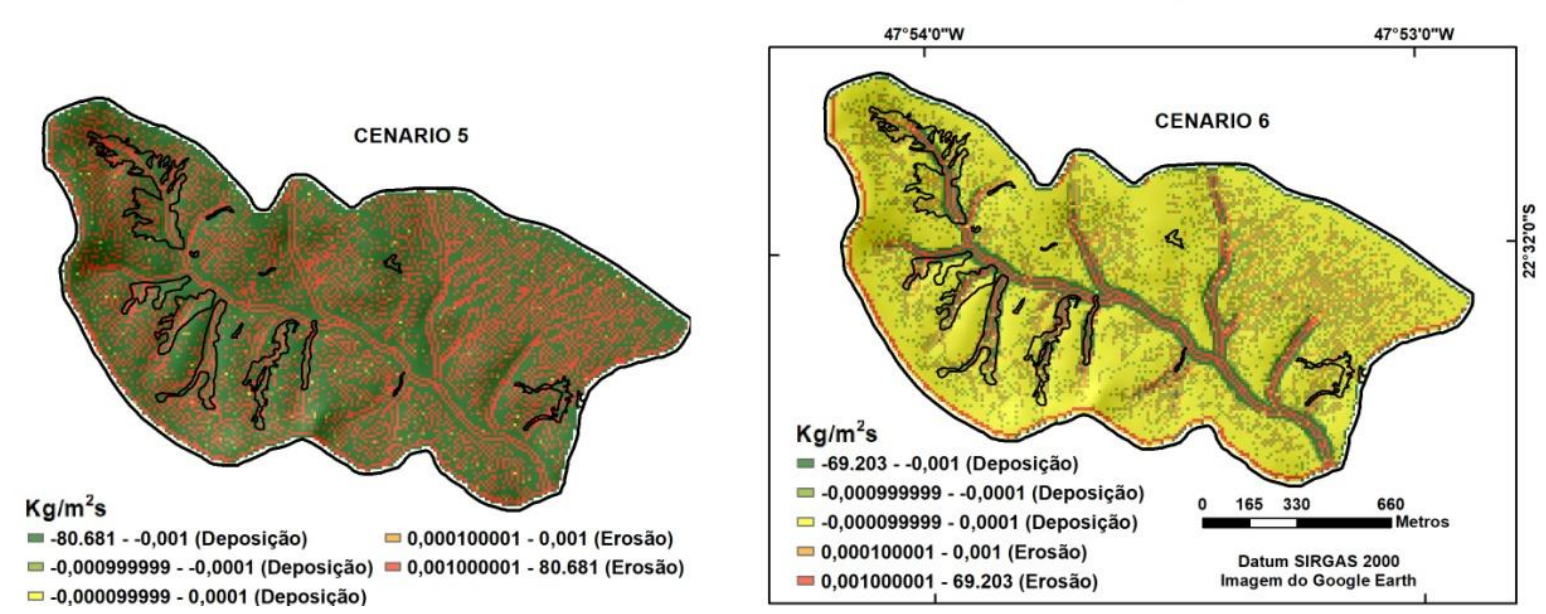

Figura 5: Distribuição espacial do balanço da erosão (valores positivos) e de deposição (valores negativos) para os cenários 1 a 6 com a sobreposição das feições erosivas de Daniel e Vieira (2015). Elaborada pelos Autores.

Nos cenários 5 e 6 notou-se uma mudança em relação aos cenários anteriores, como um aumento nos valores positivos (erosão), que alcançaram $80,68 \mathrm{~kg} / \mathrm{m}^{2} \mathrm{~s}$ acompanhados de uma ampliação espacial destas áreas pela bacia (Figura 5). Tais mudanças, nesses dois últimos cenários, podem estar associadas à exclusão do parâmetro Tensão de Cisalhamento, ao maior valor da taxa de excesso de precipitação $(190 \mathrm{~mm} / \mathrm{h})$ e ao valor máximo atribuído a taxa de infiltração $(12 \mathrm{~mm} / \mathrm{h})$ e a permeabilidade $(0,00488 \mathrm{~cm} / \mathrm{s})$ (Tabela 2). Somase também a exclusão do coeficiente da capacidade de desprendimento e do coeficiente da capacidade de 
transporte de sedimentos (Cenário 6). A sensibilidade desses dois últimos parâmetros, na comparação com o Cenário 5, praticamente não apresentou influência nos resultados.

O modelo SIMWE foi amplamente utilizado em bacias hidrográficas com atividades agrícolas na região do Vale do rio Douro em Portugal. Fernandes et al. (2015) e Fernandes et al. (2017) avaliaram a suscetibilidade à erosão linear utilizando tal modelo, que após a validação estatística, os resultados do SIMWE foram considerados moderados. De acordo com os autores, a erosão está relacionada principalmente aos processos de escoamento linear, se concentrando preferencialmente em áreas onde a profundidade da água assume valores médios e elevados. Os autores destacam, entretanto, que algumas voçorocas foram classificadas pelo modelo como processos deposicionais. Resultado este que pode ter sido causado pela associação com a morfologia original do terreno, alterada pela construção de patamares e terraços e ausência da vegetação.

Também em uma bacia com atividades agrícolas, Carvalho (2019) utilizou o modelo SIMWE para identificação e validação de ravinas e quantificação de valores de erosão, utilizando MDEs construídos antes e depois de um evento de grande magnitude. $\mathrm{O}$ autor concluiu que a sobreposição entre as feições modeladas com as feições em campo apresentou, de fato, valores positivos, confirmando a eficácia do modelo SIMWE na previsão de áreas erosivas.

Nesta pesquisa, grande parte das feições erosivas está situada em setores identificados pelo modelo com valores positivos (erosão), embora 3 feições ( $n^{\circ} 6,10$ e 13) (Figura 4) não foram identificadas nos cenários 1 a 4, as quais classificadas como áreas de deposição (valores negativos). Os cenários 5 e 6, além de identificarem as feições $\mathrm{n}^{\circ}$ 6,10 e 13 com valores positivos (erosão), também registraram áreas com suscetibilidades erosivas (valores positivos) em setores nos quais não tiveram feições erosivas mapeadas, no entanto, a partir das validações de campo verificou-se a ocorrência de sulcos erosivos.

A correlação quantitativa entre as feições erosivas e os intervalos do balanço da erosão e deposição do Cenário 5 demonstrou que $42,31 \%$ da área $\left(\mathrm{m}^{2}\right)$ das feições erosivas estão situadas nas classes de erosão (valores positivos) e 57,69\% estão situadas em locais com processos de deposição (valores negativos) (Tabela 3). Aliados às observações em campo e à sobreposição das feições erosivas mapeadas com os cenários erosão/deposição do modelo SIMWE, os dados revelaram que a presença de canais intermitentes, curvaturas côncavas, o uso e o manejo do solo associado ao solo exposto e pastagem são fatores condicionantes na formação dos sulcos erosivos. Tal circunstancia demonstra que o SIMWE pode ser aplicado em região tropical e obter resultados satisfatórios, justificativa associada à acurácia do modelo em identificar setores suscetíveis da área de estudo, sobretudo os cenários 5 e 6.

Tabela 3: Correlação entre as feições erosivas e os intervalos do balanço da erosão e deposição (Cenário 5).

\begin{tabular}{c|c|c}
\hline Classes & Feições Erosivas $\left.\mathbf{( m}^{\mathbf{2}}\right)$ & Feições $(\boldsymbol{\%})$ \\
\hline (A) $-80,68-(-0,001)$ - (Deposição) & 71068 & 57,36 \\
\hline (B) $-0,001-(-0,0001)$ - (Deposição) & 344 & 0,28 \\
(C) -0,0001 - 0,0001 - (Deposição) & 66 & 0,05 \\
(D) $0,0001-0,001$ - (Erosão) & 359 & 0,29 \\
(E) $0,001-80,68$ - (Erosão) & 52064 & 42,02 \\
\hline Total & $\mathbf{1 2 3 . 9 0 1}$ & $\mathbf{1 0 0}$ \\
\hline
\end{tabular}

Fonte: Elaborada pelos Autores.

Em relação à distribuição espacial das feições erosivas associadas aos fluxos de sedimentos e hidrológicos superficiais (Figuras 6 e 7), observou-se que os maiores valores acompanham os fluxos concentrados (afluentes e a drenagem principal). Ressalta-se que o cenário 6, em que foram desconsiderados os parâmetros de tensão de cisalhamento, coeficiente de transporte de sedimentos e o coeficiente de capacidade de desprendimento, apresentou uma distribuição espacial semelhante aos demais cenários, porém os valores do fluxo de sedimentos foram muito menores (valor máximo de $1,59 \mathrm{~kg} / \mathrm{m} . \mathrm{s}$ ) em relação aos demais cenários (entre $325 \mathrm{~kg} / \mathrm{m} . \mathrm{s}$ e $4.682 \mathrm{~kg} / \mathrm{m} . \mathrm{s}$ ) observação que demonstra que o fluxo de sedimentos do cenário 6 foi praticamente nulo devido à exclusão dos parâmetros citados.

A sobreposição com as feições erosivas permitiu verificar que uma grande parte delas está situada justamente nos setores que apresentaram maiores valores de fluxos de sedimentos e hidrológicos superficiais. Resultado este corroborado pelos trabalhos de Rodrigues (1982) e Santoro (1991) que identificaram alta intensidade dos escoamentos superficial e subsuperficial em locais com registros frequentes de ravinas e 
voçorocas. Fernandes et al (2017), a partir da aplicação do modelo SIMWE na região do Vale do rio Douro em Portugal, identificaram que as feições erosivas estão associadas às classes média e alta dos fluxos e concentração de sedimentos. Os autores também explicam que no fundo da bacia registrou-se um grande número de feições erosivas que pode estar associado aos baixos valores de infiltração e, portanto, onde estão situadas, sobretudo as altas classes dos fluxos superficiais.

As áreas com maiores valores de intensidade de fluxo prevista pelo SIMWE coincidem com a presença de Neossolos Quartzarênicos que, hidrologicamente, podem gerar maior saturação em períodos chuvosos e, consequentemente, maior capacidade erosiva (YAMANOUTH, 2003; SANTANA et al. 2007). Destaca-se os Cenários 5 e 6 que apresentaram os maiores valores de fluxo superficial associados à alta taxa de excesso de precipitação (190 mm/h); valores maiores do coeficiente de Manning (0.035n); da taxa de infiltração (12 $\mathrm{mm} / \mathrm{m})$ e da permeabilidade $(0.00488 \mathrm{~cm} / \mathrm{s})$. Tais fatores, conforme Kirkby e Chorley (1967) potencializam a suscetibilidade erosiva quando associado à maior fragilidade litológica e à curvatura côncava.
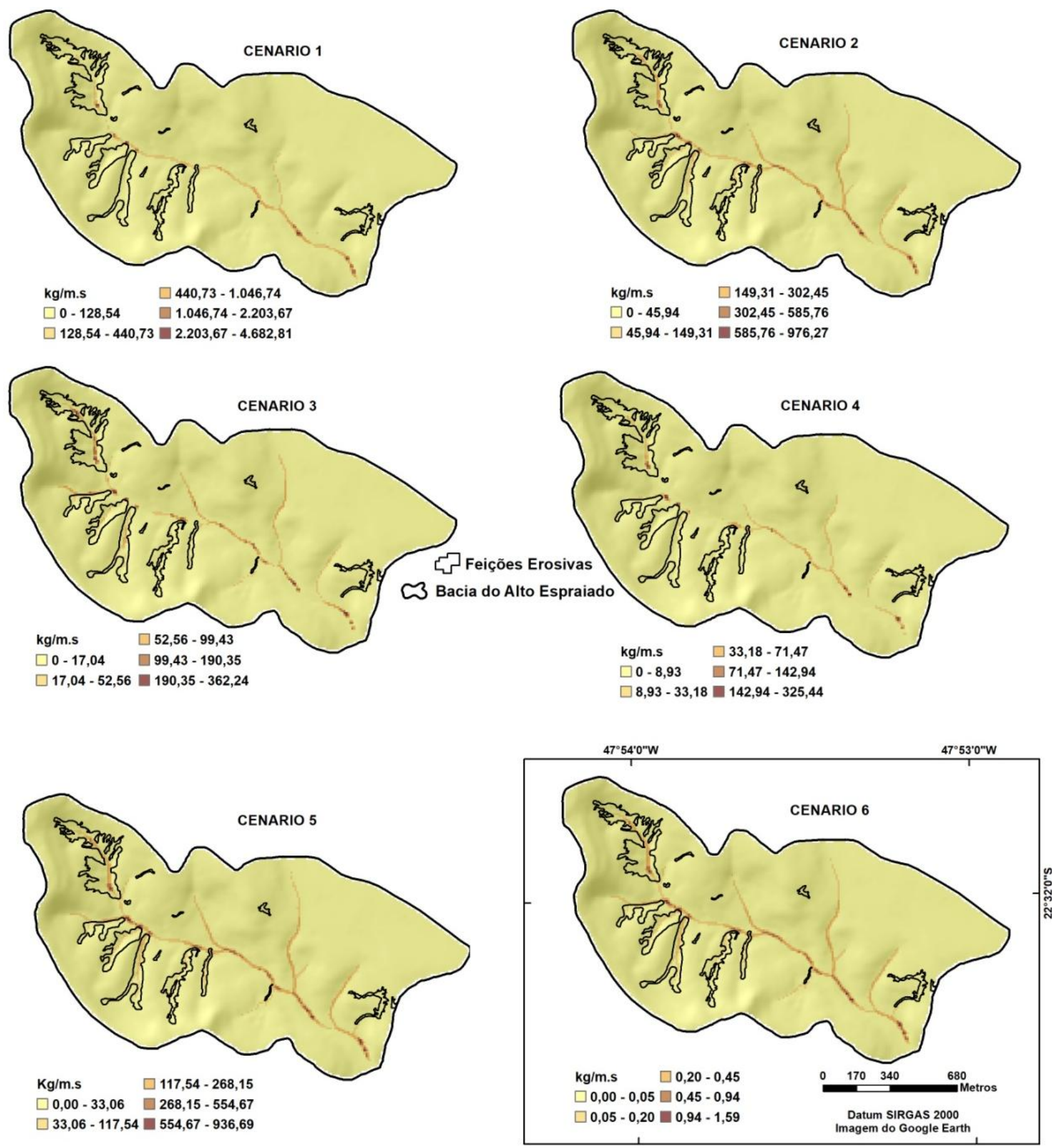

Figura 6: Distribuição espacial dos fluxos de sedimentos entre os cenários 1 a 6 com a sobreposição das feições erosivas de Daniel e Vieira (2015). Elaborada pelos Autores. 

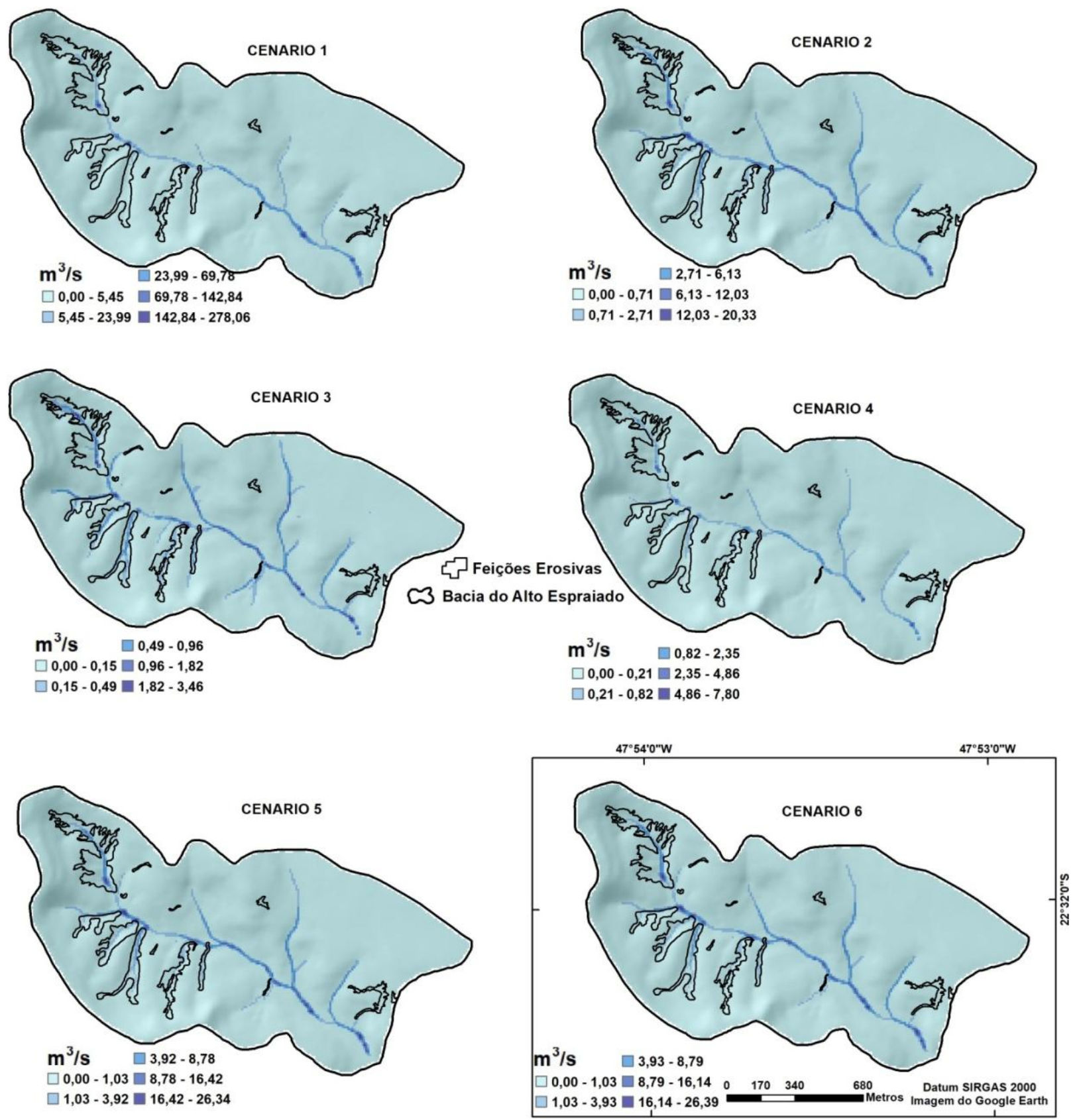

Figura 7: Distribuição espacial dos fluxos superficiais entre os cenários 1 a 6 com a sobreposição das feições erosivas de Daniel e Vieira (2015). Elaborada pelos Autores.

\section{Conclusões}

- A aplicação do modelo SIMWE na bacia do alto Espraiado demonstrou viabilidade de seu emprego para ambiente tropical, pois a maioria das feições erosivas mapeadas está situada justamente nos setores que o modelo classificou como processo erosivo (valores positivos). Tal situação demonstra a sensibilidade e a identificação de áreas suscetíveis, as quais também caracterizam pelo registro dos maiores valores de fluxos de sedimentos e hidrológicos superficiais.

- O parâmetro Tensão de Cisalhamento possui grande sensibilidade e influência na elaboração e análise dos produtos finais, sendo que a sua exclusão nos cenários 5 e 6 demonstrou maior sensibilidade do SIMWE em relação ao diagnóstico das áreas suscetíveis e correlacionada com o mapeamento.

- Destaca a sensibilidade do SIMWE nos cenários 5 e 6 em setores da média encosta que diagnosticaram suscetibilidade erosiva, e que não tinham sido mapeadas, mas validada em campo com a presença de processos erosivos iniciais (sulcos erosivos).

- O modelo SIMWE, utilizado com maior freqüência em climas temperados, pode ser uma importante ferramenta para avaliação, diagnóstico e prognóstico da suscetibilidade em paisagens sob condições climáticas mais úmidas. 


\section{Agradecimentos}

Os autores agradecem o apoio financeiro do CNPq, por meio do Projeto 870227/1997 e Processo 167550/2017-9 pela bolsa de estudo, como também o Programa de Pós-Graduação em Geografia Física da Universidade de São Paulo (USP). Agradecem também a Prefeitura de São Pedro (SP), aos proprietários rurais, ao Dr. Carlos Bateira e a Dra. Joana Filipa Costa Fernandes ambos da Universidade do Porto/Portugal, ao Geógrafo Msc. José Eduardo Bonini e a Graduanda em Geografia Camilla Pereira Capella. Também expressam seus agradecimentos aos Editores e Revisores da Revista do Departamento de Geografia pelas críticas e sugestões.

\section{Referencias}

AB'SABER, A.N. Regiões de circundesnudação pós-cretácea, no planalto brasileiro. Boletim de Geografia. São Paulo, n.1. p. 03 - 21, 1949.

ALMEIDA FILHO, G.S.; COSTA, S.B.; HELLMEISTER JUNIOR, Z.; CORSI, A.C. Situação da erosão hídrica linear no Estado de São Paulo, BR. In: ABGE, CONGRESSO DA SOCIEDADE DE ANÁLISE DE RISCO LATINO-AMERICANA, 3, São Paulo, Anais, 7 p, 2016.

AMORIM, R.S. Avaliação dos Modelos de Predição da Erosão Hídrica USLE, RUSLE e WEPP para condições edafoclimáticas brasileiras. Tese. Programa de Pós-graduação em Engenharia Agrícola, Universidade Federal de Viçosa, 2004.

AVANZI, J.C.; SILVA, M.L.N; CURI, N.; MELLO, C.R.; FONSECA, S. Calibração e aplicação do modelo MUSLE em uma microbacia hidrográfica nos Tabuleiros Costeiros brasileiros. Revista Brasileira de Engenharia Agrícola e Ambiental, v.12, n.6, p.563-569, 2008.

BERTONI, J.; LOMBARDI NETO, F. Conservação do solo. $8^{a}$ edição. São Paulo: Ícone, 2012, 335 p.

CAPELLA, C.P.; VIEIRA, B.C.; DANIEL, E. Análise do papel da chuva na formação dos processos erosivos lineares em São Pedro (SP). 13 Simpósio Nacional de Geomorfologia, Juiz de Fora, 2021.

CARVALHO, F.H. Uso do modelo SWAT na estimativa da vazão e da produção de sedimentos em bacia agrícola do Cerrado brasileiro. Dissertação. Faculdade de Agronomia e Medicina Veterinária, Universidade de Brasília, 2014.

CARVALHO, J.P.D.S. Modelação de ravinas pelo SIMWE e validação com recurso a MDE de elevação resolução: Região Demarcada do Douro, Quinta do Bonfim. Dissertação. Faculdade de Letras, Universidade do Porto, Portugal, 2019.

CIIAGRO. Centro Integrado de Informações Agrometeorológicas. Balanço Hídrico Semanal do Município de São Pedro (SP). Período de 08/07/2002 a 31/10/2019. Disponível em: http://www.ciiagro.sp.gov.br/ciiagroonline/Listagens/BH/LBalancoHidricoLocal.asp. Acesso em $14 \mathrm{de}$ Janeiro de 2021.

CONTI, J. B. Condições Climáticas da Região de Águas de São Pedro (SP). Caderno de Ciências Da Terra, Instituto de Geografia, Universidade de São Paulo, n.11, 15p, 1971.

DANIEL, E.; VIEIRA, B.C. A evolução das feições erosivas na bacia do córrego Espraiado, São Pedro (SP). Boletim Goiano de Geografia (online), v.35, n.2. p.339-359, 2015. https://doi.org/10.5216/bgg.v35i2.37435

DANIEL, E.; VIEIRA, B.C.; MARTINS, T.D. Implicações do uso do solo na ocorrência de feições erosivas em São Pedro (SP). Derbyana, 42: e751, 2021. https://doi.org/10.14295/derb.v42.751.

EMBRAPA, Empresa Brasileira de Pesquisas Agropecuárias. Sistema brasileiro de classificação de solos. 2 ed. Rio de Janeiro, 412p,1999. 
FERNANDES, E.N. Sistema inteligente de apoio ao processo de avaliação de impactos ambientais de atividade agropecuárias. Viçosa, MG: UFV, 1997. 122p. Tese (Doutorado em Ciência Florestal), Universidade Federal de Viçosa, 1997.

FERnANDES, J. Modelação de Processos Erosivos no Alto Douro Vinhateiro: o caso de estudo da Quinta de S. Luiz. Dissertação. Faculdade de Letras, Universidade do Porto, Portugal, 2014.

FERNANDES, I.; BATEIRA, C.; SOARES, L.; OLIVEIRA, A.; FARIA, A.; HERMENEGILDO, C.; TEIXEIRA, M.; MOURA, R.; GONÇALVES, J. Aplicação Do Modelo SIMWE na definição de áreas suscetíveis à erosão linear: Estudo de caso na região demarcada do Douro (RDD). VII Congresso Nacional de Geomorfologia, 2015.

FERNANDES, J., BATEIRA, C., SOARES, L., FARIA, A., OLIVEIRA, A., HERMENEGILDO, C., MOURA, R., GONÇALVES, J. SIMWE model application on susceptibility analysis to bank gully erosion in Alto Douro Wine Region agricultural terraces. Catena, n.153, p.39-49, 2017. http://dx.doi.org/10.1016/j.catena.2017.01.034

FERREIRA, M.D. Análise da evolução dos processos erosivos acelerados em áreas urbanas e das técnicas de controle e recuperação, córrego do Tucum, São Pedro (SP). Dissertação. Escola de Engenharia de São Carlos, Universidade de São Paulo, São Carlos, 191p, 2004.

FLANAGAN, D. C.; NEARING, M. A. USDA - Water Erosion Prediction Project: Hillslope Profile and Watershed Model Documentation. NSERL Report $\mathbf{N}^{\mathbf{0}}$ 10, National Soil Erosion Research Laboratory, West Lafayette, Indiana, USA, 1995.

FLANAGAN, D.C.; GILLEY, J.E.; FRANTI, T. G. WATER EROSION PREDICTION PROJECT (WEPP): Development history, model capabilities, and future enhancements. American Society of Agricultural and Biological Engineers. Mississippi, EUA, v.50(5), p.1603-1612, 2007.

FURQUIM, S. A.C. Interações entre modelo e solo no transecto do Espraiado - São Pedro/SP. Dissertação de Mestrado. Departamento de Geografia. Faculdade de Filosofia, Letras e Ciências Humanas, Universidade de São Paulo, São Paulo, 146p, 2002. https://doi.org/10.11606/D.8.2002. tde-01082005$\underline{105035}$

GOMES, D.M. Mapeamento Geotécnico para análise das feições erosivas concentradas na bacia do ribeirão do Meio, São Pedro (SP), na escala 1:20.000. Dissertação. Escola de Engenharia de São Carlos, Universidade de São Paulo, São Carlos, 268p, 2002.

GRASS, Geographic Resources Analysis Support System. Software, Version 7.2. Open-Source Geospatial Foundation, 2017.

HAO, H.X., WANG, J.G., GUO, Z.L., HUA, L. Water erosion processes and dynamic changes of sediment size distribution under the combined effects of rainfall and overland flow. Catena 173, 494 - 504, 2019. https://doi.org/10.1016/j.catena.2018.10.029

HENGL, T. Finding the right pixel size. Computers and Geosciences. n.32, p.1283-1298, 2006. http://dx.doi.org/10.1016/j.cageo.2005.11.008

HOFIERKA, J.; KNUTOVÁ, M. Simulating spatial aspects of a flash flood using the Monte Carlo method and GRASS GIS: a case study of the MaláSvinka Basin (Slovakia). Open Geosci, v.7, p.118-125, 2015. http://dx.doi.org/10.1515/geo-2015-0013

HUTCHINSON, M.F. Calculation of hydrologically sound digital elevation models. Paper presented at Third International Symposium on Spatial Data Handling at Sydney, Australia, 1988.

KAWAKUBO, F.S.; OLIVEIRA, D.; MORATO, R.G. Contribuição da análise de superfície de tendência para caracterização morfológica de uma topossequencia situada na região de São Pedro (SP).Geografia. Rio Claro, v.31, n.3, p.571-585, 2006. 
KIRKBY, M. J.; CHORLEY, R. J. Throughflow, Overland Flow and Erosion. Bulletin of the International Association of Scientific Hydrology, 12, 5-21, 1967.

KOCO, S. Simulation of gully erosion using the SIMWE model and GIS. Landform Analysis, v.17, p. 8186, 2011.

LEITE, A.P.P. Aplicação de Metodologias para o cálculo de erosão (USLE E WEPP) em uma sub-bacia hidrográfica do rio Cobres. Dissertação. Faculdade de Engenharia, Departamento de Engenharia Civil e Arquitetura, Universidade da Beira Interior, Covilhã, 2009.

MA, X.; ZHAO, C.; ZHU, J. Aggravated risk of soil erosion with global warming - A global meta-analysis. Catena, v.200, 105129, 2021. https://doi.org/10.1016/j.catena.2020.105129

MARK, D. M. "Network Models in Geomorphology." Modelling Geomorphological Systems, ed. M. G. Anderson. New York: John Wiley. 73 - 97, 1988.

MATHIAS, D. T. Implicações Geomorfológicas das Alterações do Escoamento Superficial pela Urbanização: Análise dos Processos Erosivos na bacia hidrográfica do córrego Tucunzinho (São Pedro-SP). Revista Brasileira de Recursos Hídricos, v.18 n.4, p.101-113, 2013. Disponível em: $<$ http://hdl.handle.net/11449/135257.

MINGOTI, R. Efeitos da espacialização da cobertura florestal e da profundidade dos solos na produção de água de uma bacia hidrográfica. Tese. Escola Superior de Agricultura Luiz de Queiroz, Universidade de São Paulo, Piracicaba, 171p. 2012.

MITASOVA, H., W. M. BROWN, D. M. JOHNSTON, B. SAGHARLAN, L. MITAS, AND M. ASTLEY. GIS tools for erosion/deposition modeling and multidimensional visualization. In Interpolation, rainfallrunoff, visualization. Part IV: Processed based erosion simulation for spatially complex conditions and its applications to installations, report for U.S. Army Construction Engineering Research Laboratory, p.133, University of Ilinois at Urbana Champaing, 1997.

MITAS, L.; MITASOVA, H. Distributed soil erosion simulation for effective erosion prevention. Water resources research, v. 34, n. 3, p. 505-516, 1998. https://doi.org/10.1029/97WR03347

MITASOVA H., BARTON M., ULLAH I., HOFIERKA J., HARMON, R.S. "GIS Based Soil Erosion Modeling", In Treatise on Geomorphology, Edited by John F. Shroder, 3, San Diego: Academic Press, p.228-258, 2013. http://dx.doi.org/10.1016/B978-0-12-374739-6.00052-X

NEARING, M.A.; JETTEN, V.; BAFFAUT, C.; CERDAN, O.; COUTURIER, A.; HERNANDES, M.; LE BISSONNAIS, M.H.; NICHOLS, J.P.; NUNES, C.S.; RENSCHLER, C.S.; SOUCHERE, V.; VAN OOST, K. Modeling response of soil erosion and runoff to changes in precipitation and cover. Catena, n.61, p. 131154, 2005. https://doi.org/10.1016/j.catena.2005.03.007

NETELER, M.; MITASOVA, H. Open Source Gisa Grass Gis Approach. Third Edition. The International Series in Engineering and Computer Science: v. 773, 406p, Springer, New York, 2008. https://doi.org/10.1080/13658816.2010.512275

PEREIRA, J.S. Avaliação das perdas de solos por erosão laminar na área de influencia da UHE Amador Aguiar I. Dissertação. Programa de Pós Graduação em Geografia, Instituto de Geografia, Universidade de Uberlândia, p.167, 2014.

PINTO, C. S. B. Modelagem das respostas hidrológicas as mudanças de uso e cobertura da terra na bacia do Barro Branco/São José de Ubá (RJ). Universidade Federal do Rio de Janeiro, 2014.

RENARD, K. G., FOSTER, G. R., WEESIES, G. A., McCOOL, D. K., YODER, D. C. Predicting soil erosion by water: A guide to conservation planning with the Revised Universal Soil Loss Equation (RUSLE). U.S. Department of Agriculture, Agriculture Handbook no. 703, 404 pp, 1997. 
RODRIGUES, J.E. Estudos dos fenômenos erosivos acelerados, boçorocas. Tese. Escola de Engenharia de São Carlos, Universidade de São Paulo, São Carlos, 162p, 1982.

SANCHEZ, M. C. Os municípios de São Pedro e Charqueada: aspectos de sua geografia agrária. Tese. Faculdade de Filosofia, Ciências e Letras, Rio Claro, 135 p, 1969.

SANCHEZ, M. C.. Contribuição ao conhecimento das bases naturais dos municípios de São Pedro e Charqueada (SP). Notícia Geomorfológica, v.11, 21-22, 1971.

SANTANA, N.M.P.; CASTRO, S.S.; STONE, L.F.; SILVA, S. C. Chuvas, erosividade, erodibilidade, uso do solo e suas relações com focos erosivos lineares na alta bacia do rio Araguaia. Sociedade \& Natureza, 19(2), 103-121, 2007. https://doi.org/10.1590/s1982- 45132007000200007

SANTORO, J. Fenômenos erosivos acelerados na região de São Pedro (SP): estudo da fenomenologia, com ênfase geotécnica. Dissertação. (Mestrado em Geociências e Análise Ambiental), Instituto de Geociências e Ciências Exatas, Universidade Estadual Paulista, Rio Claro, 140 p, 1991.

SANTORO, J.; FULFARO, V.J. Estudos Geotécnicos em Boçoroca na Cidade de São Pedro, São Paulo (SP). Revista Instituto Geológico, São Paulo, 17(1/2), p.55-62, 1996. http://dx.doi.org/10.5935/0100$\underline{\text { 929X.19960003 }}$

SANTOS, G.G.; NORI, P.G.; OLIVEIRA, L.F.C. Chuvas intensas relacionadas a erosão hídrica. Revista Brasileira de Engenharia Agrícola e Ambiental, v.14, n.2, p. 115-123, 2010.

SILVA, A.M. Aplicações de técnicas de geoprocessamento no estudo das relações entre os processos erosivos e sedimentológicos de bacia hidrográfica. Tese. Escola de Engenharia de São Carlos, Universidade de São Paulo, São Carlos, 269p, 1999.

TARBOTON, D.G., BRAS, R.L.; RODRIGUES-ITURBE, I. On the Extraction of Channel Networks from Digital Elevation Data. Hydrological Processes, Chichester,v.5, n.1, p. 81-100, 1991.

TERRA FOTO, S.A. Governo do Estado de São Paulo, Secretaria de Economia e Planejamento, Plano Cartográfico do Estado de São Paulo. Carta Topográfica, Escala 1:10.000, São Pedro I (SF-23-Y-A-IV-1NO-B), 1979.

WARREN, S.T. Digital Terrain Modeling and Distributed Soil Erosion Simulation/Measurement for Minimizing Environmental Impacts of Military Training. US Army Corps of Engineers Construction Engineering Research Laboratories, 1998.

WILLIAMS, J. R. Sediment routing for agricultural watersheds. In: Water Resources Bulletin. Proceedings of the AWRA, Minneápolis, 11 v., nº 5, Oct. 1975. p. 965-974, 1975.

WILLIAMS, J. R. Sediment-yield prediction with Universal Equation using runoff energy factor. In: Present and prospective technology for predicting sediment yield and sources. Oxford: USDA. ARS-S-40, p.244-252, 1975.

WISCHMEIER, W. H. \& SMITH, D. D. Predicting rainfall-erosion losses from cropland east of the Rocky Mountains-guide for selection of practices for soil and water conservation. Washington, D.C.: USDA (Agriculture Handbook, 282), 1965.

WISCHMEIER, W. H.; SMITH, D. D. Predicting rainfall erosion losses: a guide to conservation planning. Washington, DC: USDA, (Agriculture handbook, 537), 1978.

YAMANOUTH, G.R.B. Avaliação dos processos erosivos e das técnicas de controle e reabilitação bacia do córrego do Espraiado - São Pedro (SP). Dissertação. (Mestrado em Geotecnia). Escola de Engenharia de São Carlos, Universidade de São Paulo, São Carlos, 177 p, 2003. https://doi.org/10.11606/D.18.2016.tde-11042016-161906 
